Marquette University

e-Publications@Marquette

College of Education Faculty Research and

Publications

Education, College of

$12-1-2020$

\title{
The MOVE Framework: Meanings, Observations, Viewpoints, and Experiences in Processes of Social Change
}

Seamus A. Power

University of Copenhagen

Gabriel Velez

Marquette University, gabriel.velez@marquette.edu

Follow this and additional works at: https://epublications.marquette.edu/edu_fac

Part of the Education Commons

\section{Recommended Citation}

Power, Seamus A. and Velez, Gabriel, "The MOVE Framework: Meanings, Observations, Viewpoints, and Experiences in Processes of Social Change" (2020). College of Education Faculty Research and Publications. 544.

https://epublications.marquette.edu/edu_fac/544 
Marquette University

e-Publications@Marquette

\title{
Education Faculty Research and Publications/College of Education
}

This paper is NOT THE PUBLISHED VERSION.

Access the published version via the link in the citation below.

Review of General Psychology, Vol. 24, No. 4 (December 1, 2020): 321-344. DOI. This article is (C) American Psychological Association and permission has been granted for this version to appear in ePublications@Marquette. American Psychological Association does not grant permission for this article to be further copied/distributed or hosted elsewhere without express permission from American Psychological Association.

\section{The MOVE Framework: Meanings, Observations, Viewpoints, and Experiences in Processes of Social Change}

Séamus A. Power

University of Copenhagen, Copenhagen, Denmark

Gabriel Velez

Marquette University, Milwaukee, WI

\begin{abstract}
Social psychologists are often criticized for failing to capture the dynamic nature of psychological processes. We present a novel framework to address this problem. The MOVE framework contends that to comprehend complex, contradictory, and divergent patterns of thought, affect, and behavior within changing, real-world contexts, it is necessary to undertake ecologically valid research that is attentive to the lived experiences and meaning-making processes of culturally embedded individuals over time. A focus on meanings, observations, viewpoints, and experiences is essential for social psychological research that holistically captures how people construct, understand, respond,
\end{abstract}


position, and act over time within changing social, economic, and political contexts. To illustrate the utility of our proposition, we draw on classic social psychological studies and multimethod fieldwork during a period of rapid social and political change in Colombia during the peace process (2012-2017). We argue the MOVE framework has the potential to advance psychological understandings of, and contributions to, individuals embedded in real, dynamic social and political contexts. We discuss the implications of this extended social psychological paradigm for advancing psychological science.

\section{Keywords}

ecological validity, social change, qualitative methods, quantitative method, replication crisis

\section{Introduction}

Social psychologists play a key role in understanding how the human mind processes and functions on individual, group, and national levels. However, there is often a disjunction between the methods employed by psychologists to study social phenomena and the multifaceted, dynamic, and temporal realities of life. Specifically, static analyses devoid of cultural, historical, economic, legal, and political context are often applied to complex psychological phenomena that unfold over time (Bartlett, 1930, 1958; Gergen, 1973; Hammack, 2008, 2011a, 2011b; Henriques, 2013; Moghaddam, 2002, 2013, 2018; Reicher, 2017; Reicher \& Haslam, 2012; Shweder, 1991, 2003; Smedslund, 2009; Tateo \& Valsiner, 2015; Wagoner, 2017; Wagoner et al., 2012). Yet, the very nature of these contexts is dynamic, and this inherently influences cognitive, social, and behavioral processes. Periods of rapid sociopolitical change present even greater flux for individuals, groups, and nations. Therefore, social psychological methodologies and models must be attentive to this fluctuation to provide insights into how people within these contexts process, think about, and respond within these changing social realities on individual and group levels.

In this article, we detail the MOVE framework as a broad conceptual and methodological model to guide multilevel psychological study of the mind amid dynamic sociopolitical contexts. Importantly, what we propose would entail changes in the culture, education, and research of psychology as a discipline, and we argue this is necessary to address deeply embedded issues the field currently faces. The MOVE framework proposes that addressing meanings, observations, viewpoints, and experiences improves the ecological validity of social psychological science and can be a foundation for research more attuned to social context and its changing nature over time. First, to situate and motivate the novel framework within the broader literature in social psychology, we examine both previous and recent methodological crises and the conceptualizations of psychological science these crises originated from. We discuss the contemporary replication crisis-instigated by the Open Science Collaboration (2015) revelation that a minority of studies published in top tier psychology journals replicate-as well as a related crisis in the 1970s concerning a lack of ecological validity in psychological research (Faye, 2012; Hammond, 1998). We detail lessons from these crises for the conceptual and methodological development of social psychological research. We propose an expansion of the social psychological paradigm in light of the limitations of experimental approaches to richly capture processes in dynamic sociopolitical contexts. In this first section, we draw on historical roots of psychology to motivate our framework as an innovative approach to addressing issues currently critical to the field of social psychology (Cabell \& Valsiner, 2014). 
Second, we outline a framework to overcome the current and pervasive problems with social psychological research by addressing meanings, observations, viewpoints, and experiences. Applying our framework, a more holistic, dynamic, and complete picture of social psychological phenomena can be understood and described at different levels of analysis. Each of our four components is interrelated and critical to our proposal to advance social psychological science. In this second section, we use examples from classical social psychological research to illuminate the utility of the theoretical framework.

Third, to highlight the importance and utility of the MOVE framework, we discuss its application to contemporary social psychological research. Specifically, we provide an example of transdisciplinary, multimethod research, in Colombia from 2012 to 2017. During this time, the Colombian government finalized peace accords with the largest armed revolutionary group. The agreement was divisive within the country, and discourses about the responsibility of young Colombians to be peace builders permeated the education sector and civil society more broadly. We use a multifaceted, multimethod study of young people's unfolding conceptualizations of peace to illustrate the limitations of using traditional social psychological methods in isolation and to highlight the utility and novelty of the MOVE framework to comprehend social psychological perspectives of unfolding sociopolitical phenomena.

\section{Motivation: Overcoming Crises in Social Psychology}

The timing and utility of our methodological review and new proposal is motivated by the current state of social psychological science. Amassing scientific knowledge through systematic and replicable experimental findings is the hallmark of good science (Chambers, 2017; Open Science Collaboration, 2012; Reyna, 2004). However, recent attempts to replicate some high-profile experiments, most notably in social psychology, have failed to a larger degree than expected (Baker, 2016; Open Science Collaboration, 2015). This incongruence between expectations of replications and the reality of nonconfirming results has, according to some, lead to the "replication crisis" in psychology. Although there have been efforts to address these concerns, on the whole, discussions about this crisis and the underlying issues have been fragmented (Rodgers \& Shrout, 2017; Schmidt, 2009; Zwaan et al., 2018).

Multiple reasons have been given for the replication crisis in psychology. Some psychologists have lamented the oversampling of undergraduate students from U.S. universities and the generalization of findings from this atypical group across populations and cultures (Arnett, 2008; Henrich et al., 2010; Sears, 1986). When replications of these results are attempted on non-WEIRD (western, educated, industrial, rich, and democratic) samples, the results can be incongruent. This is because although there are psychological universals, these do not necessarily manifest uniformly across time and cultures and these nuances can be missed with applying experimental procedures in one context to another (Henrich et al., 2010; Norenzayan \& Heine, 2005; Shweder, 2010). Other researchers have criticized scientific standards regarding $p$-values. Fearing too many false positives-a problem exasperated by $p$-hacking (selectively reporting data and analyses) and HARKing (hypothesizing after the results are known; see Nelson et al., 2018)-some social scientists have argued for lowering the acceptable $p$-value threshold or refocusing on adequate statistical power (Benjamin et al., 2018; Szucs \& loannidis, 2017; Muthukrishna \& Henrich, 2019). Overall, the crisis has led to many points of contention: questions about when and if replication studies should be expected to fail; debates over 
the informational value provided by replications in a field that values novel and counter intuitive findings; issues concerning status and reputation of experiments and (failed) replications of them; debates about the importance of direct or conceptual replications; and disagreements over deciding when a study has or has not been replicated (Rodgers \& Shrout, 2017; Schmidt, 2009; Zwaan et al., 2018).

Most recent attempts to address the replication crisis have focused on changes within the dominant experimental paradigm (e.g., Munafò et al., 2017; van't Veer \& Giner-Sorolla, 2016). We support our colleagues' efforts to develop social psychological science who argue and advocate for the importance of preregistering theoretically driven hypotheses, making data available on the open science framework, increasing diversity and size of samples, and publishing replicated findings. In addition to these endeavors, our argument in this article is the social psychological paradigm also needs to be expanded to improve our understanding of individuals and groups in unfolding sociopolitical contexts. We interpret the replication crisis as demonstrating the methodological limits of the current focus in social psychology. Specifically, we argue this narrow view-predominated by laboratory and online studies using quantitative methods within a causal framework-does not provide for fruitful and reliable study of complex social psychological processes and phenomena in ecologically meaningful ways within dynamic contexts.

We argue part of this disjuncture is about a lack of productive integration across methods. In an earlier conceptual model, along with two colleagues, we addressed this by proposing the SAGE model of social psychological research as a guide to think holistically about psychological research as a multimethod process (Power et al., 2018). We highlighted incongruencies on an ontological level between historical conceptualizations of complementary forms of social psychological approaches and contemporary orientations. We proposed a mixed-methods model to combine qualitative and quantitative methods and overcome this disjunction on a practical level. We argued for the importance of a Synthetic model, where qualitative methods were Augmentative to quantitative methods, where they were Generative of hypotheses where predictions could be tested experimentally, and where qualitative methods could be used to examine Experiences that evade experiment reductionism. We argued this SAGE model was a wise approach to conducting social psychological research to more holistically understand the individual in context. From a similar epistemological stance that psychological phenomena require complex, multimethod approaches, the MOVE framework identifies, and aims to solve, a different issue in social psychological research.

That is, the MOVE framework outlines a conceptual and practical way for social psychological researchers to more effectively study the complexity of phenomena amid dynamic social and political contexts. One novel contribution of our proposed framework is to suggest its utilization will lead to a greater understanding of why experiments will, or will not, replicate either directly or conceptually, when researchers account more strongly the ecological validity of their studies. Greater understanding and recognition of the context in which participants perform experiments-and in which experimental results are interpreted-will allow for richer and more broadly applicable research findings, which might aid replications either directly or indirectly by laying bare boundary conditions that is what results are expected in what particular cultural time and context. This will aid our understanding of what findings might be universal and others that are particular to time and place. Another novel 
contribution of the MOVE framework is to go beyond static experimental analyses of complex, unfolding, and sociopolitical phenomena. In unfolding, rapidly changing sociopolitical realities, psychological phenomenon cannot be researched and analyzed with singular snapshots in controlled environments. Our proposed model offers concrete guides for more productive social psychological research in dynamic contexts.

\section{Lessons From the Past}

We draw support for this argument from the historical roots of psychology. In certain respects, the modern crisis involving experiments reveals a disconnect with the discipline's foundations. Wundt (1897) in Outlines of Psychology conceptualized a two-tier psychological science. Experimental lab psychology-focusing on manipulating variables and studying outcomes-was to be used to determine causal inference on basic perception. The second tier of research was meant to examine complex psychological functioning on a cultural level using complementary methodologies. Nevertheless, Wundt's vision of psychology was never realized. In the early 20th century, the psychoanalytic approach to studying the human mind prized an exploration of experiences and meaning, but the advent of behaviorism in the United States decontextualized the mind (Cabell \& Valsiner, 2014; Harré \& Moghaddam, 2015; Moghaddam, 2013; Rozin, 2009). A few decades later, the cognitive revolution reintroduced the mind to psychology as it moved beyond the reductionist and incomplete behaviorist "stimulus followed by response" model. It served to put the complexities of the culturally situated and mediated mind back into the psychological frame (Bruner, 1990; Shweder, 2012). Nevertheless, the dominant conceptualization of the mind as a computer again decontextualized the study of social psychological phenomena. The mind was seen as an isolated entity, capable of being examined independent of the diverse contexts in which it was embedded.

Keeping with an historical perspective to ground the MOVE framework, psychology has been in crisis before. Social psychologists in the 1970s struggled with deep and difficult questions related to methodology, societal relevance, and theoretical and philosophical directions (see Faye, 2012). These issues were intertwined, complex, and ultimately led to efforts to bridge the gap between research and social psychological processes and phenomena in complicated and diverse contexts. Some social psychologists called for methodological pluralism to overcome limitations and advance the scopes of singular methods to investigate social scientific phenomena (Fiske \& Shweder, 1986). However, as a discipline, social psychology has moved toward a narrower attention to the individual and their responses and behaviors, leaving contextualization to sociologists and anthropologists (Faye, 2019). Pluralism is currently minimized in the field. Experimental manipulation of variables in decontextualized contexts are prized (Nisbett, 2015; Reyna, 2004).

Of course, the careful experimentation of variables is essential to social psychological research. We admire much of the groundbreaking experimental social psychological research being conducted by our colleagues. Still, the problems of experimental decontextualization have been well documented (Asch, 1952/1987; Power et al., 2018; Rozin, 2001, 2009). The availability of research participants via the internet, such as by Amazon MTurk, has led to increased opportunity to run multiple experiments and multiple iterations of the same experiment quickly and relatively cheap. These internet samples can be beneficial: They provide a source of pilot data that can be insightful for further developing studies and can increase sample size to bolster statistical power and reliability. However, like all 
samples, online participants are limited, and not simply because of questions of diversity (Gamblin et al., 2017; Goodman et al., 2013). One key problem is the separating of participants from ecological valid contexts. Social psychological research often assumes the generalizability of universal principles derived from limited samples. Although psychological universals exist, they do not manifest uniformly across the world (Cassaniti \& Menon, 2017; Norenzayan \& Heine, 2005; Henrich et al., 2010; Markus \& Kitayama, 1991; Shweder, 1991, 2003; Wang, 2013). Generalizing from limited samples-particularly those that are abstracted from ecological contexts - can be perilous because people in the real-world process and respond to stimuli in relation to their particular ecology and understandings of it (i.e., emotions, thoughts, feelings, behaviors, representations, memories, and imaginings). A second key issue with experiments is they are typically snapshot, when social phenomena are inherently dynamic. Documenting one aspect of a process is inherently incomplete. An erroneous assumption of many experimental social psychologists is that combining studies can reveal an accurate explanation of social psychological processes and phenomena. In contrast, a holistic social psychology assumes the sum is greater than the parts: Unfolding processes cannot be holistically explained using experimental procedures. Advanced statistical methods and longitudinal studies are more nuanced in relation to the unfolding nature of human development, but are often disconnected from experimental work (West et al., 2004) and lack strong ecological validity (Lewin, 1943/2014; Rozin, 2001, 2009).

As social psychologists, we aim not to explain what goes on in our experiments or in numerical representations. Rather, the goal is to use experiments and quantitative data to try to understand the processes that explain what goes on in the real world. The first step in any good science is to map the phenomenon: Observe what is happening to generate sound hypotheses at the start (Reicher, 2017). But this is seldom discussed as a salient and important issue in social psychology. For example, analyses of psychology blogs revealed psychologists are concerned with the replication crisis, statistical significance, and science communication (Nicolas et al., 2019). However, what is not included is revealing about the current state of social psychological science. Issues to do with meaning-making processes, psychological observations, multiple viewpoints, and subjective experiences are neglected. They are certainly not mainstream in research, as evidenced, for example, by a content analysis of the three premier journals in psychology revealing no purely qualitative empirical studies in the year 2016 which are often used to capture social psychological processes (Power et al., 2018).

Social psychological science, and the study of unfolding social psychological processes and phenomena more broadly, can advance with an implementation of the MOVE framework. A synthetic application of mixed methods has the potential to overcome the limits of individual methods, like experimental manipulation, and generates a framework to study processes as they unfold over time.

\section{A Solution: The MOVE Framework to (Re)expand the Social Psychological Paradigm}

The four elements of the MOVE framework (meanings, observations, viewpoints, experiences) arise from a divide in the physical and social world between what is quanta and what is qualia (Shweder, 1996). The quanta world is comprised of everything that is left when the world is rid of human subjectivities. This is the world that can be studied using quantitative methods to measure, abstract, and quantify natural phenomena. In contrast, the world of qualia is the subjective world. It is the world 
humans construct, represent, reflect upon, experience, and make meaning of. Qualitative research is based in qualia; the study of concepts, cultures, interpretations, and representations are a path to understanding how humans think, feel, want, moralize, and value (Bruner, 1990; Cole, 1998; Markus \& Kitayama, 1991; Shweder, 1991, 2003; Wierzbicka, 1993). Human psychological processes and phenomena cannot be conceptualized solely through a numerical focus on objects, events, and nonsubjective processes or through decontextualized or artificially contextualized experiments in a lab. Instead, it requires an approach attentive to the individual factors-subjective realities, interpretation, and response-and social, cultural, historical, economic, political, and legal contexts that are inherently dynamic and unfolding (Asch, 1952/1987; Hammack, 2008; Power et al., 2018; Rozin, 2001, 2009; Shweder, 1991, 2003).

We argue meanings, observations, viewpoints, and experiences are four quintessential components to in-depth social psychological study in shifting sociopolitical contexts. These four components encapsulate the essence of the world of qualia and have been specifically chosen because they are critical and interconnected elements of social psychological research that have been de-emphasized. In the following sections, we discuss each element of the MOVE hypotheses in more depth for conceptual clarity. Importantly, emerging from the world of qualia, in actuality, they are simultaneously distinct, overlapping, and connected.

\section{Meanings}

The first pillar of our MOVE framework is a focus on meaning. We use meaning to refer to how individuals - as agentic members of groups, communities, societies, and nations-interpret and construct norms, ideas, and attitudes in relation to their lived experiences in their social, cultural, and political contexts (Blumer, 1986; Bruner, 1990; Hammack \& Cohler, 2009; Wagoner, 2009). We propose that social psychological research can refocus on meanings in two ways. First, a focus on meaning suggests attentiveness to subjects' understandings and perspectives. Concepts, definitions, and acts cannot be assumed to be consonant with prevalent frameworks (or those from the researcher's cultural perspective), but must be coherent with people's own understandings (Atran \& Norenzayan, 2004; Shweder, 1991, 2003). Second, a variety of methods should be used in combination, with each informing the others and contributing to more flexible and intricate models of social psychological phenomena (Power et al., 2018). This element is inherently related to the first; to be attentive to diverse perspectives and understandings, a researcher must employ diverse methods.

These concrete steps support ecological validity by drawing attention to the psychological processes and orientations as people navigate and create their social worlds. A focus on meaning stands in contrast to current trends and dominant approaches in social psychology. It is important to expand beyond decontextualized experiments to better understand phenomena and psychological response as situated in lived, dynamic realities. Without multiple methods focused on triangulating individuals' understandings of their worlds, researchers may fall into the trap of believing they are studying one phenomenon while their subjects hold a completely different mental framework that changes over time (Campbell \& Fiske, 1959; Denzin, 2012; Fiske \& Shweder, 1986; Henriques, 2013; Schiff, 2017; Shweder, 2010; Tateo \& Valsiner, 2015). We propose observational methods are important to comprehend shifts in meaning-making processes that occur in shifting sociopolitical contexts. 
Meaning making is inherently a process; it occurs over time as ecological contexts change, groups form and reform, and individuals develop along ontological life courses. We hypothesize that a refocus on meaning-making processes that unfold over time would prioritize how people make sense of their worlds, their experiences, and themselves (Bruner, 1990; Spencer et al., 1997; Tateo \& Valsiner, 2015).

A concrete demonstration of the importance of meaning comes from early work in psychology, but maintains clear reverberations in modern times. Discussing a famous study differentiating populations in New Guinea with Western samples based on an experiment involving skin pricks, E. B. Titchener (1916) pointed out the Papuans may simply have been interpreting what they were being asked to do differently than the Westerners. He argued these respondents had a qualitatively different experience of the task itself, rather than simply a different response to it. Shweder (2010) makes the connection between Titchener's critique and the issue with cross-cultural claims based on the Ultimatum Game as an experimental paradigm. Shweder raises the question of how certain populations interpret the basic framework of "playing a game with an anonymous other." Social networks built on gift-giving as the means to status, for example, may drive different responses and challenge the very construction of what the stimuli is. In other words, "evidence of psychological differences between cultural groups may simply (although significantly) demonstrate that different stimulus situations produce different responses" (Shweder, 2010, p. 109). This cultural critique-levied by others as well-underscores the variance in how the task itself is interpreted (Chuah et al., 2007; Henrich, 2000). As demonstrated in Titchener's historical analysis, and this modern application, the very meaning of a stimuli may be in doubt without an attentiveness to how individuals and groups understand and think about the world.

\section{Observations}

Meaning is rooted in individuals' lived experiences of social context, which in turn motivates our second element, observation. By observation, we refer to the systematic study of people within their natural contexts and everyday lives over prolonged periods of time (Geertz, 1973; Gorman et al., 2005; Rai \& Fiske, 2010). We propose observation take a primary role in social psychological science in two concrete manners. First, we suggest ethnographic observation be more prominent in research projects to describe and analyze meaning-making in diverse contexts over time. Second, observation should be used to inform, and make sense of, quantitative and experimental tests of theory-driven hypotheses (see Muthukrishna \& Henrich, 2019). These two applications provide the opportunity for greater ecological validity in investigations into the relationship between people and context, as well as how, and why, this relationship changes over time. A focus on observation can drive research questions and methodological choices, as well as generate data for analysis. Used in this way, observation offers a check of external and ecological validity of quantitative and experimental findings.

In our proposed re-expansion of the social psychological paradigm, observing entails scientific and open-minded inspection and study of actual people and their experiences, rather than applying preconceived frameworks or assumptions to social psychological phenomena of interest. Careful and scientifically conducted observation can help identify dissonance between research paradigms, questions, hypotheses, and predictions, on one hand, and people's own experiences and meaning making on the other hand. It can highlight paradoxes, contradictions, patterns, and new behaviors that then can be studied through appropriate instruments (Geertz, 1973; Kagan, 2012; Rai \& Fiske, 
2010; Shweder, 1997). This observational documentation can guide methodological and analytic choices, while also offering rich data about how people experience the world.

By including observation, we do not simply argue for more naturalistic field studies. Rather, we promote research and theory that treats individuals as agents operating within various and dynamic contexts, while highlighting the need for continued naturalistic observation as dynamic contexts develop (Cohen et al., 1996; Paluck, 2009; Paluck \& Cialdini, 2014). According to the qualia/quanta distinction outlined above, people are subjects, not objects, and should be studied as such (Power, 2014).

Again, a historical example demonstrates the utility and role of this element of our MOVE framework. Stanley Milgram's work on obedience has become a pillar of introductory psychology courses and permeated popular understandings of obedience to authority. Decades of iterations and replications have suggested individuals are capable of violent actions beyond what they imagined they would do when prodded by an authority figure. This plethora of studies has also identified cultural and contextual factors that facilitate or hamper these actions (Blass, 1999). Yet, this prolific area of inquiry began with an observation.

In "Eichmann in Jerusalem" Hannah Arendt introduced the concept of the "banality of evil" (1963). Based on her observations of the Nuremburg trials, she noted how Eichmann, who had been responsible for murdering millions of Jewish people during the Holocaust, was not a senseless monster. Instead, she observed he was simply following orders and divorced himself from personal responsibility for the genocide. By fragmenting responsibility, he separated himself from blame. Thus, the "banality of evil" concept was coined to describe this phenomenon. Following observations of the trial of Eichmann, and Arendt's conceptualization of the "banality of evil," Milgram began to wonder about questions of when and how people followed orders, which held profound implications for thinking about morality and accountability (Milgram, 1974). Although Milgram ultimately began to explore these questions in a laboratory setting, the motivation began with careful observation of the real world.

\section{Viewpoints}

Viewpoints, the third element of our proposal, is derived from a number of theoretical perspectives. We draw on cultural psychological theory emphasizing how individuals and cultures co-constitute each other (Cole, 1998; Shweder, 1991, 2003), positioning theory highlighting how people within these cultures position themselves in relation to others (Harré \& Moghaddam, 2003), and social representations theory illustrating how individuals represent their social worlds from their unique positions (Moscovici, 1961/2008). With viewpoints, we prioritize attentiveness to two aspects of social life: The many varied ecologies in which individuals-research participants and researchers-are embedded, as well as how positions and social representations change over time (Bronfenbrenner, 2009; Gillespie \& Cornish, 2010). We assert true ecological validity in social psychological research necessitates multiple methods and studies to attend to, meaningfully capture, and triangulate this diversity and mutability (Denzin, 2012; Henriques, 2013; Munafò \& Davey-Smith, 2018).

Viewpoints is connected to meaning, but they are separate pillars of our proposal. Although meaning draws attention beyond outcomes and quantitative measurements to include social psychological 
processing and interpretation, viewpoints is about recognizing and addressing the range of perspectives and social contexts. Viewpoints situates meaning and lived realities within cultural and historical frameworks. Subjects and researchers have positionalities tied to their identities, societies, social groups, and lived experiences. Therefore, viewpoints complements meaning by proposing an awareness and attentiveness to this human and ecological diversity.

This third pillar can boost validity by enriching understandings of social psychological processes at different levels of analysis. Validity is a multifaceted concept that has been discussed in scientific research for almost a 100 years and in psychology for nearly as long (Hathaway \& McKinley, 1943; Remmers et al., 1927). Generally, it refers to measuring what is purported to be measured (Brewer, 2000; Lewin, 1943/2014), but also incorporates many elements researchers must consider, such as internal, external, construct, concurrent, and predictive. Viewpoints can bolster ecological validity through attention to the social positions and representations of diverse groups of people. In the early stages, attention to varied viewpoints can help in the conceptualization and design of studies. Awareness of various social worlds and the corresponding stimuli can drive testable research hypotheses (Shweder, 2010). This same focus can guide the development of experiments and protocols that are valid within the setting they are applied. Viewpoints can thus boost ecological validity by leading to findings that are more applicable and reliable in actual, lived realities (Brewer, 2000; Lewin, 1943/2014).

The object of study, however, is not the only challenge to validity involved in a narrow approach. Psychological researchers must think about the viewpoints embedded in methodological approaches and ourselves as researchers in varied, interconnected contexts (Bronfenbrenner \& Ceci, 1994; Langdridge, 2008). Each researcher carries their own disciplinary lens, personal experiences, and cultural frames. No research can be absolutely devoid of epistemological positioning, but a lack of awareness to this may omit other important perspectives. A focus on viewpoints also entails reflection on the ways that a researcher's own stance, background, and approach may affect validity. In suggesting this focus, we recognize there is much discussion and debate currently over whether research is inherently value neutral or not (Duarte et al., 2015). We wish simply to raise attention to the positionalities of researchers and promote greater attention to what diverse viewpoints offer psychological study.

For example, in a classic study of the diffusion of knowledge over time, Moscovici traced how the new and increasingly popular phenomenon of psychoanalysis was understood, experienced, and represented by different groups across French society (Moscovici, 1961/2008). He was interested in individual and group viewpoints concerning this unfamiliar phenomenon. The research showed how different social groups and mass media constructed and reformed knowledge about psychoanalysis as it spread throughout French society given their unique sociocultural values and beliefs. Catholics conceptualized psychoanalysis as a supplement to, but not a replacement of, confession. However, a sexualized representation of humanity was rejected as incompatible with teachings of the Catholic church. In contrast, communists completely rejected psychoanalysis because they viewed it as being on the wrong side of their dichotomized worldview. French liberals, including liberal media, accepted the novel practice, as it was congruent with their ideologically expansive worldview. 
By investigating how social groups made the unfamiliar familiar, Moscovici provides a social psychological model for considering how people made meaning of, and ultimately experience (or not), a novel phenomenon from their particular sociocultural viewpoints. In fact, partly from this work, Moscovici (1981) developed a theory of social representations to frame the iterative coconstruction of individuals and collective understandings based on affiliations and group dynamics. As such, considering viewpoints with ecologically valid methods (qualitative and quantitative) that followed a process of diffusion over time allowed for an understanding of how knowledge in society is transformed. This classic investigation provides an example congruent with the MOVE framework because it illustrates a multimethod, ecologically valid, way to examine an unfolding social phenomenon on individual and group levels over a prolonged period of time.

\section{Experiences}

The final element is experiences, by which we refer to the thoughts, feelings, and actions of people. In other words, we use experience in reference to the agency of individuals amid changing social, political, and historical contexts (Geertz, 1973; Shweder, 1996). Experiences are the integrative result of meaning making processes, observations about the social world, and people's articulation and embodiment of viewpoints. To this end, stimuli-such as a social event, context, or moment-the meaning attributed to it, and individual's response to it all encompass their "experience" as a holistic phenomenon encompassing more than just their fully conscious processing of lived realities. This definition takes a more holistic view than focusing just on the stimuli, a behavioral response, or affective outcomes. Experience is complementary to meaning, observations, and viewpoints. Meaning highlights interpretation and psychological response should be considered, observations entails how people position themselves in the world, and viewpoints incorporates multiple perspectives that must be considered. Bringing this all together, experience draws attention to contextualizing meaning making within dynamic individual and social trajectories.

With experience, we propose two elements to refocus social psychological research. First, it is critical to move beyond deterministic frames that directly link contexts to outcomes (Kagan, 2012; Moghaddam, 2013; Valsiner \& Chaudhary, 2017). Our framework prioritizes exploring and understanding what comes between contexts and outcomes; that is, how changing stimuli are received, integrated, and responded to. Second, social psychological research should be situated within ontological and historical development to truly understand the ways that events, trends, dynamics, and narratives in social contexts influence individual and collective psychologies (Bruner, 1990; Hammack, 2008, 2012; Shweder, 1991, 2003).

Measuring experience can be challenging. Simply asking individuals to describe experience ignores evidence that memory and consciousness can only partially explain social psychological phenomena (e.g., Velmans, 2000). This is why we situate experience as part of our broader, integrative model. We argue experience is a necessary focus of social psychological research and understandings, and it is through observation and attentiveness to diverse viewpoints and how people make meaning of their social worlds-as studied with multiple measures-that we can understand lived-experiences.

System justification theory, for example, asserts people who live in dictatorships generally do not revolt because they have a need to maintain the status quo to ensure individual psychological stability. An impressive oeuvre of correlation and experimental studies support this theory (for a review, 
see Jost, 2019). Yet, recent ethnographic work challenges this assumption. Research in dictatorships presents an alternative perspective based on understanding people's lived experiences in these settings (Moghaddam, 2013, 2016). People are not necessarily driven to justify unfair systems. They are often acutely aware of injustices and unfairness within these systems. Ethnographic research and other empirical evidence reveal they do not revolt because of fear (Adra et al., 2019). Citizens in dictatorships are afraid of punishment to them or their families in the form of torture, imprisonment, beatings, arrest, or death at the hands of secret police. An alternative account of this social psychological phenomenon was developed by understanding the lived experiences of people over prolonged time periods through observing and studying their viewpoints and meaning making in complex sociopolitical contexts using ecologically valid methods.

\section{The Integrated MOVE}

Although we have separated the four elements of our MOVE framework to provide detail and rationale for each, as the examples from social psychology show, the elements are also interdependent. All four are deduced from a conceptualization of the world of qualia: the world of subjectivities, cultures, interpretations, and representations. A focus on meaning should be tied to ecologically valid measurements and studies rooted in observation. A better grasp of meaning making processes from the viewpoints of diverse samples facilitates understanding lived experience. Observation provides a tool for understanding the complexity, variance, and nuance of how context is experienced, as well as the researcher's own phenomenology. Multiple methods can be used to test and check ideas generated via naturalistic observation when applied in synthesis with this reflection on the researcher's cultural frameworks (Henriques, 2013). Finally, we can better understand people's individual and collective experience of stimuli by observing how people-across various cultures and perspectives - make meaning, respond, and behave over time (Tateo \& Valsiner, 2015).

Our MOVE framework thus comes together: capturing the dynamic and ecologically embedded nature of human minds would be strengthened by research projects that explore lived experience that is attentive to multiple viewpoints and address individual's processes of psychological meaning through using naturalistic observation at various stages of the process.

MOVE is not simply a rearticulation of mixed methods, but rather an epistemological orientation with concrete steps that can extend the current social psychological research paradigm. Engaging with the broad concept of research that is sensitive to meanings, observations, viewpoints, and experiences, it is possible to generate finer grained, ecologically valid hypotheses. These hypotheses in turn can lead to specific predictions about social psychological phenomena, which may be empirically tested experimentally, and in conjunction with other methods, to develop social psychological theories to understand unfolding processes of sociopolitical change.

We propose the MOVE framework can serve as a guide for addressing multiple challenges, such as studying the dynamic interaction between individuals and cultures and having methods that are flexible, integrative, and capable of addressing sociopolitical change (Bruner, 1990; Shweder, 1991, 2003; Valsiner \& Chaudhary, 2017). Specifically, MOVE should be implemented as a guiding framework: Its elements can be drawn on in conceptualizing holistic research projects, as well as developing research questions, generating testable hypotheses, planning methodologies for data collection and analysis, and interpreting results. Each of these stages requires ecological validity if the 
broader research is to meaningfully address dynamic social phenomena that unfold over time.

The MOVE framework is thus an approach to research, with the four elements playing out concretely in orientations, choices, and motivations in the social psychological study of inherently dynamic phenomena. The framework can be applied to how individuals, groups, and nations understand and experience dramatic sociopolitical change.

\section{MOVE in Practice: The Case of Colombia}

An example of how the MOVE framework can be applied to effectively study a dynamic context is the peace process in Colombia from 2012 to 2017. During this time, the Colombian government and the oldest armed guerrilla group, the Revolutionary Armed Forces of Colombia-People's Army (FARC-EP), negotiated 300-page accords to end over five decades of armed conflict. Although the peace talks garnered the international spotlight, the process also involved concerted efforts to change the broader narrative of Colombia as a violent country. A focus of these efforts was educating and calling on young people to be active peace builders. Young Colombians received messages and classroom lessons urging them to be politically and socially active in building peace, but placing the success of this societal effort on their shoulders (Sánchez Merteens, 2017). Many frameworks were presented to these young people about how they should think about peace. Furthermore, the peace process divided the electorate when put to a vote, which highlighted the role of young people-and their conceptualizations of peace-in creating and maintaining prolonged peace in Colombia. Research examined, using multiple methods, as the peace process was unfolding, how Colombian youth in their localized contexts thought about peace and themselves (Velez, 2019; Velez et al., 2019). The research exemplified integrating attention to meaning (of young Colombians and as part of their psychological development), observation (of the changing social context, education about peace, protests and peace movements), viewpoints (incorporating multiple methods and perspectives, while epistemologically maintaining an attentiveness to Velez's own positionality), and experience (of peace, efficacy as citizens, and societal change). Overall, the Colombia example and the questions it raises broadly represent a common and important challenge for social psychologists: How can we best study the complexities of individuals in context as sociopolitical phenomena unfold in real world settings, especially ones of broad societal change?

In line with our MOVE framework, the investigation in Colombia began with prolonged and informal observation, which was then further developed into scientific study. As a teacher during the beginning of the peace process, Velez engaged in naturalistic observations: discussions with fellow teachers, witnessing peace-related school and district events, and talking with students. This raised questions about how young people themselves were thinking about conflict, their country's historical and future trajectory, and themselves. These initial observations were further developed with more structured observation. Velez returned numerous times to participate and observe peace education curriculum development, and youth activities and demonstrations related to peace process. These experiences, across multiple years, were intentionally chosen to understand and learn about peace discourses and education across various sectors and actors in Colombia. All in all, this set up demonstrates the iterative and flexible nature of MOVE: Continued observation and integration of various viewpoints as macrolevel events develop is necessary to effectively situate the investigation of the social psychological phenomenon of question. 
The MOVE framework guided the research itself as a multimethod process that took place over several years. Velez began by exploring how youth were experiencing Colombia's peace process and broader social and political trajectory. A first part of this puzzle involves understanding broader changes in youth attitudes. To this end, Velez drew on international data sets to examine changing youth civic attitudes in Colombia, which found evidence that even as young Colombians became more disillusioned with the traditional political system, they were not detaching from political engagement (Velez \& Knowles, in press). This finding provided a background context for understanding how youth constructed meanings of peace; the quantitative data set is static, yet revealing, and a more dynamic framework was needed to investigate how this finding, coupled with the naturalistic observations, related to young Colombians' development as citizens.

The quantitative analysis provided a broader glimpse of how young people were experiencing this context, but in line with MOVE, more richly understanding this experience required attention as well to meaning and diversity within viewpoints. At two time points-just before the accords were finalized and after they had begun to be implemented-more than 320 adolescents across Colombia were interviewed about the peace process, their thinking about peace, and how they understood their possible contributions to peace. In a semiexperimental procedure, participants were also presented with excerpts from the government's proposed peace education curriculum, and asked to respond (Velez, 2019, in press). Finally, a smaller subset of participants was interviewed 3 years in a row. This additional consideration over time helped understand the how young people's ideas about peace might shift with their own developmental trajectory, as well as changes in the peace process. Themes in their answers were also compared with a discourse analysis of government speeches and documents prior to the interview data collection to situate young Colombians' meaning making within a broader discursive context.

Following MOVE, this approach allowed not only to explore abstract meaning making, but how it connected to lived experience. The research also involved attention to a diversity of viewpoints, as well as the multiple conceptions young people may be cognitively processing at the same time (e.g., an abstract idea, their interpretation of lived experiences, an understanding of their own efficacy). Focusing on how young Colombians experienced the peace process and discourses demonstrated their own ideas reflected broader discourses, but then were adapted to fit their own understandings of themselves and their society. Addressing meaning making in relation to lived experiences, drawing on this element of MOVE demonstrated they held nuanced ideas about peace that influenced their civic action and changed dependent on context. Speaking abstractly, they often mirrored government discourses saying peace began internally in each person, but as other contexts, conversations, and responses were considered, they demonstrated changing ideas about its possibility and their own efficacy to contribute to peace based on external, systemic issues like corruption, violence in other places, and their own developmental challenges.

An application of the MOVE framework in the Colombian case demonstrates an attentiveness to people's meaning (conceptual understandings and cognitive processing), observations (of the various educational contexts and movements about peace), viewpoints (analyses of various forms of data, from multiple sources and socioeconomic groups, over a prolonged period of time), and how adolescents more broadly experienced the peace process and social context with a focus on the ways 
they related their own identities to it. Although this example is particular in its historical, cultural, social, economic, and legal context, the application of the MOVE framework highlights the importance for social psychological conceptualizations of thoughts, feelings, viewpoints, and behaviors in unfolding sociopolitical contexts.

\section{Conclusion}

We propose the MOVE framework to comprehend complex patterns of thought and behavior within dynamic, real-world contexts. It is grounded in the assertion that it is necessary to undertake ecologically valid research of unfolding social psychological processes, particularly during periods of rapid societal and political change. Meanings, observations, viewpoints, and experiences lie at the heart of understanding social psychological processes at individual, group, and national levels. The decontextualization of research and the reification of the experiment as the dominant means of inquiry in social psychology are limited (Asch, 1952/1987; Rozin, 2001, 2009). In isolation, paradigmatic approaches may be fundamentally at odds with the dynamic and unfolding experiences of individuals in their social contexts. Social science research must employ approaches capable of studying movement and processes of change, and we propose our MOVE framework to address this challenge in social psychology.

With MOVE, we push for social psychology to move beyond a limited orientation that compartmentalizes different parts of the human experience to different disciplines. Under such a framework, social psychology should focus on stimuli, response, and behavior as rooted within an individual. These processes are decontextualized, leaving the day-to-day world and social context to sociologists and anthropologists to investigate (Faye, 2012, 2019). We argue, however, that to truly study and understand the human psyche-inherently and deeply interconnected with cultural and social context (Bronfenbrenner, 2009; Moscovici, 1981; Shweder, 1991)-in an ecologically valid way, social psychology must face head on the dynamic nature of lived experiences. MOVE provides a framework to try accomplish this goal.

There are distinct advantages to studying psychological reactions over time amid changing political and social dynamics by using locally meaningful, ecologically valid, multiple methods that are close to observed thoughts, feelings, and actions of people in their contexts. First, it describes and explains the phenomena of interest as it develops over time. Second, it helps generate more precise hypotheses and predictions that can be further understood on a general level using ecologically valid experiments. Failure to replicate ought to inform the scopes of generalizability and/or boundary conditions of the phenomena. This is because social psychological experiments occur within specific ecological contexts. A greater understanding of the parameters of these contexts will inform our understand of why, or why not, an experimental finding does or does not replicate, either directly or conceptually. Third, it provides a framework for conceptualizing social psychological processes. The Colombian case, for example, informs our comprehension of how young people in transitional contexts relate to peace and related discourses as they make meaning based on their various viewpoints and experiences. But more broadly, it illustrates how individuals, as part of social groups and nations, comprehend and act in shifting economic and sociopolitical conditions. We invite other researchers to utilize our framework, to test whether it indeed provides greater insight in to the ecologically valid study of how individuals in context understand and make meaning of their sociocultural and political worlds. 
Importantly, however, we are not proposing simply a rejection of quantification and laboratory studies. Instead, the framework is a broader, more holistic orientation that addresses the limits of the experimental method (as highlighted by the contemporary and previous replication crisis) and quantitative-only approaches. The application of the laws of experimentation and use of quantitative methodologies are essential (Chambers, 2017; Reyna, 2004). Yet, it would be inappropriate to solely apply these to study the qualia world of subjectivities (Shweder, 1996). The world of meaning making, observation, multiple viewpoints, and lived experiences is often difficult to understand solely in a laboratory (Power, 2011; Shweder, 1996, 2010). This world is inherently dynamic: people, societies, political landscapes, discourses, and environments constantly change. Laboratory experiments often offer snapshots, and quantitative studies may miss the dynamic ways that people respond based on context, continuity, and change in their sociocultural, political, and economic contexts. They are important methodologies, but cannot be the only ones used in isolation.

An application of the MOVE framework has implications for social psychological science. The replication "crisis" is one of the discipline's major issues at the moment (Baker, 2016; Open Science Collaboration, 2015; Schmidt, 2009). The MOVE framework has the potential to develop social psychological science by advancing approaches to understand processes; that is, through attention to meaning-making and lived experiences in context and as changing over time via observations and multimethod research projects. It also prioritizes reflection on researchers' own schema and positionality, as well as incorporating broad and diverse samples. These elements increase ecological validity, which in turn reveals boundary conditions of experimental investigations (i.e., an understanding of the scopes and limits of a context in which an experimental procedure is likely to replicate or not), or demonstrations of social phenomena (Paluck \& Cialdini, 2014). In this way, the MOVE framework illustrates the scopes and limits of the experimental method to describe, comprehend, or predict the dynamics of social psychological phenomena. Replication, when integrated with other methods and used in relation to MOVE, can be one part of triangulation to test and develop social psychological theories (Denzin, 2012; Munafò \& Davey-Smith, 2018).

The MOVE framework is not without its challenges, and these should not be understated. Conducting research that focuses on meanings and experiences that unfold in shifting contexts is time consuming, requires expertise in diverse methods, may be difficult to publish, has implications at multiple levels of analysis, and may produce incongruent or contradictory results. Decontextualization and reification of the experiment in psychology presents obstacles to studying social psychological processes over time. These issues are deeply embedded in the field: in training, in research, in what is prized in publication, and in underlying philosophical orientations. In the face of these difficulties, there are potential benefits for social psychologists to understand and utilize contextualized and integrative approaches. To engage with the big issues facing the world-inequalities and injustices, economic hardship, conflict, populism - we need significant paradigmatic advances to bridge the gap between our research and the impact it has. Although we have proposed MOVE to advance social psychological research, we also believe in the importance of greater discussion across disciplines to construct more nuanced and insightful frameworks of the mind. We believe our proposed framework can extend social psychology and even bridge disciplines, and we welcome further extension and development both within psychology and across the social sciences. 


\section{Acknowledgements}

The authors would like to thank Alex Gillespie, David Sherman, Richard Shweder, and Martha Van Haitsma for their feedback on an earlier draft of this manuscript.

\section{Declaration of Conflicting Interests}

The author(s) declared no potential conflicts of interest with respect to the research, authorship, and/or publication of this article

\section{Funding}

The author(s) received no financial support for the research, authorship, and/or publication of this article.

\section{References}

\begin{tabular}{|c|}
\hline $\begin{array}{l}\text { Adra, A., Harb, C., Li, M., Baumert, A. (2019). Predicting collective action tendencies among Filipina } \\
\text { domestic workers in Lebanon: integrating the social identity model of collective action and } \\
\text { the role of fear. Group Processes \& Intergroup Relations. Advance online } \\
\text { publication. https://0-doi-org.libus.csd.mu.edu/10.1177/1368430219885180 }\end{array}$ \\
\hline Arendt, H. (1963). Eichmann in Jerusalem: a report on the nanality of evil. Penguin Books Ltd. \\
\hline $\begin{array}{l}\text { Arnett, J. (2008). The neglected 95\%: Why American psychology needs to become less American. } \\
\text { American Psychologist, 63, 602-614. }\end{array}$ \\
\hline Asch, S. E. (1987). Social psychology. Oxford University Press. (Original work published 1952) \\
\hline $\begin{array}{l}\text { Atran, S., Norenzayan, A. (2004). Religion's evolutionary landscape: Counterintuition, commitment, } \\
\text { compassion, communion. Behavioral and Brain Sciences, 27(6), 713-730. }\end{array}$ \\
\hline Baker, M. (2016). 1,500 scientists lift the lid on reproducibility. Nature News, 533(7604), 452-454. \\
\hline Bartlett, F. (1958). Thinking: An experimental and social study. Basic Books. \\
\hline $\begin{array}{l}\text { Bartlett, F. C. (1930). Experimental method in Psychology. The Journal of General Psychology, 4(1- } \\
\text { 4), 49-66. }\end{array}$ \\
\hline $\begin{array}{l}\text { Benjamin, D. J., Berger, J. O., Johannesson, M., Nosek, B. A., Wagenmakers, E. J., Berk, R., . . . } \\
\text { Cesarini, D. (2018). Redefine statistical significance. Nature Human Behaviour, 2(1), 6-10. }\end{array}$ \\
\hline $\begin{array}{l}\text { Blass, T. (1999). The Milgram paradigm after } 35 \text { years: Some things we now know about obedience } \\
\text { to authority. Journal of Applied Social Psychology, 29(5), 955-978. }\end{array}$ \\
\hline Blumer, H. (1986). Symbolic interactionism: Perspective and method. University of California Press. \\
\hline $\begin{array}{l}\text { Brewer, M. B. (2000). Research design and issues of validity. In Reis, H. T., Judd, C. M. (Eds.), } \\
\text { Handbook of research methods in social and personality psychology (pp. 3-16). Cambridge } \\
\text { University Press. }\end{array}$ \\
\hline Bronfenbrenner, U. (2009). The ecology of human development. Harvard University Press. \\
\hline $\begin{array}{c}\text { Bronfenbrenner, U., Ceci, S. J. (1994). Nature-nurture reconceptualized in developmental } \\
\text { perspective: A bioecological model. Psychological Review, 101(4), 568-586. }\end{array}$ \\
\hline Bruner, J. (1990). Acts of meaning. Harvard University Press. \\
\hline $\begin{array}{l}\text { Cabell, K. R., Valsiner, J. (2014). Systematic systemics: Causality, catalysis, and developmental } \\
\text { cybernetics. In Cabell, K. R., Valsiner, J. (Eds.), The catalyzing mind (pp. 3-13). Springer. }\end{array}$ \\
\hline $\begin{array}{l}\text { Campbell, D. T., Fiske, D. W. (1959). Convergent and discriminant validation by the multitrait- } \\
\text { multimethod matrix. Psychological Bulletin, } 56,81-105 \text {. }\end{array}$ \\
\hline
\end{tabular}


Cassaniti, J. L., Menon, U. (Eds.). (2017). Universalism without uniformity: Explorations in mind and culture. University of Chicago Press.

Chambers, C. (2017). The seven deadly sins of psychology: A manifesto for reforming the culture of scientific practice. Princeton University Press.

Chuah, S. H., Hoffmann, R., Jones, M., Williams, G. (2007). Do cultures clash? Evidence from crossnational ultimatum game experiments. Journal of Economic Behavior \& Organization, 64(1), 35-48.

Cohen, D., Nisbett, R. E., Bowdle, B. F., Schwarz, N. (1996). Insult, aggression, and the southern culture of honor: An "experimental ethnography." Journal of Personality and Social Psychology, 70(5), 945-959.

Cole, M. (1998). Cultural psychology: A once and future discipline. Harvard University Press.

Denzin, N. K. (2012). Triangulation 2.0. Journal of Mixed Methods Research, 6(2), 80-88.

Duarte, J. L., Crawford, J. T., Stern, C., Haidt, J., Jussim, L., Tetlock, P. E. (2015). Political diversity will improve social psychological science. Behavioral and Brain Sciences, 38, Article e130.

Faye, C. (2012). American social psychology: Examining the contours of the 1970s crisis. Studies in History and Philosophy of Science Part C: Studies in History and Philosophy of Biological and Biomedical Sciences, 43(2), 514-521.

Faye, C. (2019). Social psychology. In Sternberg, R., Pickren, W. (Eds.), The Cambridge handbook of the intellectual history of psychology (pp. 318-344). Cambridge University Press. https://0doi-org.libus.csd.mu.edu/10.1017/9781108290876.013

Fiske, D. W., Shweder, R. A. (Eds.). (1986). Metatheory in social science. University of Chicago Press.

Gamblin, B. W., Winslow, M. P., Lindsay, B., Newsom, A. W., Kehn, A. (2017). Comparing in-person, Sona, and Mechanical Turk measurements of three prejudice-relevant constructs. Current Psychology, 36(2), 217-224.

Geertz, C. (1973). Interpretation of cultures. Basic Books.

Gergen, K. J. (1973). Social psychology as history. Journal of Personality and Social Psychology, 26(2), 309-320.

Gillespie, A., Cornish, F. (2010). Intersubjectivity: Towards a dialogical analysis. Journal for the Theory of Social Behaviour, 40, 19-46.

Goodman, J. K., Cryder, C. E., Cheema, A. (2013). Data collection in a flat world: The strengths and weaknesses of Mechanical Turk samples. Journal of Behavioral Decision Making, 26(3), 213224.

Gorman, G. E., Clayton, P. R., Shep, S. J., Clayton, A. (2005). Qualitative research for the information professional: A practical handbook. Facet Publishing.

Hammack, P. L. (2008). Narrative and the cultural psychology of identity. Personality and Social Psychology Review, 12(3), 222-247.

Hammack, P. L. (2011a). Narrative and the politics of identity: The cultural psychology of Israeli and Palestinian youth. Oxford University Press.

Hammack, P. L. (2011b). Narrative and the politics of meaning. Narrative Inquiry, 21(2), 311-318.

Hammack, P. L., Cohler, B. J. (Eds.). (2009). The story of sexual identity: Narrative perspectives on the gay and lesbian life course. Oxford University Press.

Hammond, K. R. (1998). Ecological validity: Then and now [Unpublished manuscript]. http://www.albany.edu/cpr/brunswik/notes/essay2.html

Harré, R., Moghaddam, F. (Eds.). (2003). The self and others: Positioning individuals and groups in personal, political, and cultural contexts. Greenwood. 
Harré, R., Moghaddam, F. (2015). Positioning theory and social representations. In Sammut, G., Andreouli, E., Gaskell, G., Valsiner, J. (Eds.), The Cambridge handbook of social representations (pp. 224-233). Cambridge University Press.

Hathaway, S. R., McKinley, J. C. (1943). The Minnesota Multiphasic Personality Inventory (Rev. ed., 2nd Printing). University of Minnesota Press.

Henrich, J. (2000). Does culture matter in economic behavior? Ultimatum game bargaining among the Machiguenga of the Peruvian Amazon. American Economic Review, 90(4), 973-979.

Henrich, J., Heine, S., Norenzayan, A. (2010). The weirdest people in the world? Behavioral \& Brain Sciences, 33, 61-83.

Henriques, G. (2013). Evolving from methodological to conceptual unification. Review of General Psychology, 17(2), 168-173. https://0-doi-org.libus.csd.mu.edu/10.1037/a0032929

Jost, J. T. (2019). A quarter century of system justification theory: Questions, answers, criticisms, and societal applications. British Journal of Social Psychology, 58(2), 263-314.

Kagan, J. (2012). Psychology's ghosts: The crisis in the profession and the way back. Yale University Press.

Langdridge, D. (2008). Phenomenology and critical social psychology: Directions and debates in theory and research. Social and Personality Psychology Compass, 2(3), 1126-1142.

Lewin, K. (2014). Psychological ecology. In Gieseking, J. J., Mangold, W., Katz, C., Low, S., Saegert, S. (Eds.), The people, place, and space reader (pp. 17-21). Routledge. (Original work published 1943)

Markus, H. R., Kitayama, S. (1991). Culture and the self: Implications for cognition, emotion, and motivation. Psycho-logical Review, 98(2), 224-253.

Milgram, S. (1974). Obedience to authority: An experimental view. Tavistock.

Moghaddam, F. M. (2002). The individual and society: A cultural integration. Worth.

Moghaddam, F. M. (2013). The psychology of dictatorship. American Psychological Association.

Moghaddam, F. M. (2016). The psychology of democracy. American Psychological Association.

Moghaddam, F. M. (2018). Political plasticity and revolution. In Wagoner, B., Moghaddam, F. M., Valsiner, J. (Eds.), The psychology of radical social change: From rage to revolution (pp. 122139). Cambridge University Press.

Moscovici, S. (1981). On social representations. In Forgas, J. P. (Ed.), Social cognition: Perspectives on everyday understanding (pp. 181-209). Academic Press.

Moscovici, S. (2008). Psychoanalysis: Its image and its public. Polity Press.

Munafò, M. R., Davey-Smith, G. (2018). Robust research needs many lines of evidence. Nature News, 553, 399-401.

Munafò, M. R., Nosek, B. A., Bishop, D. V., Button, K. S., Chambers, C. D., Du Sert, N. P., . . loannidis, J. P. (2017). A manifesto for reproducible science. Nature Human Behaviour, 1(1), 1-9.

Muthukrishna, M., Henrich, J. (2019). A problem in theory. Nature Human Behaviour, 3, 221229. https://0-doi-org.libus.csd.mu.edu/10.1038/s41562-018-0522-1

Nelson, L. D., Simmons, J., Simonsohn, U. (2018). Psychology's renaissance. Annual Review of Psychology, 69, 511-534.

Nicolas, G., Bai, X., Fiske, S. T. (2019). Exploring research-methods blogs in psychology: Who posts what about whom, and with what effect? Perspectives on Psychological Science, 14, 691704. https://0-doi-org.libus.csd.mu.edu/10.1177/1745691619835216

Nisbett, R. E. (2015). Mindware: Tools for smart thinking. Farrar, Straus and Giroux. 
Norenzayan, A., Heine, S. J. (2005). Psychological universals: What are they and how can we know? Psychological Bulletin, 131(5), 763-784.

Open Science Collaboration . (2012). An open, large-scale, collaborative effort to estimate the reproducibility of psychological science. Perspectives on Psychological Science, 7(6), 657-660.

Open Science Collaboration . (2015). Estimating the reproducibility of psychological science. Science, 349, 944-951.

Paluck, E. L. (2009). Reducing intergroup prejudice and conflict using the media: A field experiment in Rwanda. Journal of Personality and Social Psychology, 96(3), 574-587.

Paluck, E. L., Cialdini, R. B. (2014). Field research methods. In Reis, H., Judd, C. (Eds.), Handbook of research methods in social and personality psychology (pp. 81-97). Cambridge University Press.

Power, S. A. (2011). On social psychology and conflict resolution. Psychology \& Society, 4(1), 1-6.

Power, S. A. (2014). Humans as objects: Processes, predictions and problems. Peace \& Conflict: Journal of Peace Psychology, 20(4), 593-594.

Power, S. A., Velez, G., Qadafi, A., Tennant, J. (2018). The SAGE model of social psychological research. Perspectives on Psychological Science, 13(3), 359-372.

Rai, T. S., Fiske, A. (2010). ODD (observation-and description-deprived) psychological research. Behavioral and Brain Sciences, 33(2-3), 106-107.

Reicher, S. D. (2017). "La beauté est dans la rue": Four reasons (or perhaps five) to study crowds. Group Processes \& Intergroup Relations, 20(5), 593-605.

Reicher, S. D., Haslam, S. A. (2012). Change we can believe in: The role of social identity, cognitive alternatives, and leadership in group mobilization and transformation. In Wagoner, B., Jensen, E., Oldmeadow, J. A. (Eds.), Culture and social change: Transforming society through the power of ideas (pp. 53-73). Information Age.

Remmers, H. H., Shock, N. W., Kelly, E. L. (1927). An empirical study of the validity of the SpearmanBrown formula as applied to the Purdue rating scale. Journal of Educational Psychology, 18(3), 187-195.

Reyna, V. F. (2004). Why scientific research? The importance of evidence in changing educational practice. In McCardle, P., Chlabra, V. (Eds.), The voice of evidence: Bringing research to classroom educators (pp. 47-58). Brookes.

Rodgers, J. L., Shrout, P. E. (2017). Psychology's replication crisis as scientific opportunity: A précis for policymakers. Policy Insights from the Behavioral and Brain Sciences, 5, 134-

141. https://0-doi-org.libus.csd.mu.edu/10.1177/2372732217749254

Rozin, P. (2001). Social psychology and science: Some lessons from Solomon Asch. Personality and Social Psychology Review, 5, 2-14.

Rozin, P. (2009). What kind of empirical research should we publish, fund and reward? A different perspective. Perspectives on Psychological Science, 4, 435-439.

Sánchez Merteens, A . (2017). Los Saberes de La Guerra: Memoria y Conocimiento Intergeneracional Del Conflicto En Colombia [Understandings of the War: Memory and Intergenerational Knowledge of the Colombian Conflict]. Siglo del Hombre Editores; Universidad Nacional de Colombia.

Schiff, B. (2017). Explorations in narrative psychology. A new narrative for psychology. Oxford University Press.

Schmidt, S. (2009). Shall we really do it again? The powerful concept of replication is neglected in the social sciences. Review of General Psychology, 13(2), 90-100. https://0-doi-

org.libus.csd.mu.edu/10.1037/a0015108 
Sears, D. O. (1986). College sophomores in the laboratory: Influence of a narrow data base on social psychology's view of human nature. Journal of Personality and Social Psychology, 51, 515530.

Shweder, R. A. (1991). Thinking through cultures: Expeditions in cultural psychology. Harvard University Press.

Shweder, R. A. (1996). Quanta and qualia: What is the "object" of ethnographic method? In Jessor, R., Colby, A., Shweder, R. A. (Eds.), Ethnography and human development: Context and meaning in social inquiry (pp. 175-182). University of Chicago Press.

Shweder, R. A. (1997). The surprise of ethnography. Ethos, 25(2), 152-163.

Shweder, R. A. (2003). Why do men barbecue? Recipes for cultural psychology. Harvard University Press.

Shweder, R. A. (2010). Donald Campbell's doubt: Cultural difference or failure of communication? Behavioral and Brain Sciences, 33(2-3), 109-110.

Shweder, R. A. (2012). Anthropology's disenchantment with the cognitive revolution. Topics in Cognitive Science, 4, 354-361.

Smedslund, J. (2009). The mismatch between current research methods and the nature of psychological phenomena: What researchers must learn from practitioners. Theory \& Psychology, 19(6), 778-794.

Spencer, M. B., Dupree, D., Hartmann, T. (1997). A phenomenological variant of ecological systems theory (PVEST): A self-organization perspective in context. Development and Psychopathology, 9(4), 817-833.

Szucs, D., loannidis, J. P. (2017). Empirical assessment of published effect sizes and power in the recent cognitive neuroscience and psychology literature. PLoS Biology, 15(3), e2000797.

Tateo, L., Valsiner, J. (2015). Time breath of psychological theories: A meta-theoretical focus. Review of General Psychology, 19(3), 357-364. https://0-doiorg.libus.csd.mu.edu/10.1037/gpr0000035

Titchener, E. B. (1916). On ethnological tests of sensation and perception with special reference to tests of color vision and tactile discrimination described in the reports of the Cambridge anthropological expedition to Torres Straits. Proceedings of the American Philosophical Society, 55(3), 204-236.

Valsiner, J., Chaudhary, N. (2017). From methodology to methods in human psychology. Springer.

Van't Veer, A. E., Giner-Sorolla, R. (2016). Pre-registration in social psychology-A discussion and suggested template. Journal of Experimental Social Psychology, 67, 2-12.

Velez, G. (2019). Conceptualized peace: A study of Colombian adolescents' meaning making and civic development [Unpub-lished Doctoral dissertation]. University of Chicago.

Velez, G. (in press. Learning peace: Adolescent Colombian's interpretations and responses to peace education curriculum. Peace and Conflict: Journal of Peace Psychology.

Velez, G., Ballesteros, A., Sánchez Merteens, A. (2019). La voz de la juventud: perspectivas juveniles sobre la guerra, la paz y la reintegración en Colombia. In Rettberg, A., McFee, E. (Eds.), Implementación del acuerdo de paz con la(s) FARC y reintegración: Un balance de la etapa temprana e implicaciones para política pública. Universidad de los Andes.

Velez, G., Knowles, R. (in press). Trust, civic self-efficacy, and acceptance of corruption among Colombian adolescents. Journal of Research in Comparative and International Education.

Velmans, M. (Ed.). (2000). Investigating phenomenal consciousness: New methodologies and maps (Vol. 13). John Benjamins. 
Wagoner, B. (Ed.). (2009). Symbolic transformation: The mind in movement through culture and society. Routledge.

Wagoner, B. (2017). The constructive mind: Bartlett's psychology in reconstruction. Cambridge University Press.

Wagoner, B., Jensen, E., Oldmeadow, J. A. (2012). Culture and social change: Transforming society through the power of ideas. Information Age.

Wang, Q. (2013). The autobiographical self in time and culture. Oxford University Press.

West, S. G., Biesanz, J. C., Kwok, O. M. (2004). Within-subject and longitudinal experiments: Design and analysis issues. In Sansone, C., Morf, C. C., Panter, A. T. (Eds.), The SAGE handbook of methods in social psychology (pp. 287-312). SAGE.

Wierzbicka, A. (1993). A conceptual basis for cultural psychology. Ethos, 21(2), 205-231.

Wundt, W. (1897). Outlines of psychology (Judd, C. H. , Trans.). Scholarly Press.

Zwaan, R. A., Etz, A., Lucas, R. E., Donnellan, M. B. (2018). Making replication mainstream. Behavioral and Brain Sciences, 41, Article e120. 